\title{
ESTUDIO DE LOS FACTORES CRÍTICOS PARA EL ÉXITO EN LA CONSTRUCCIÓN DE EDIFICACIONES EN LA CIUDAD DE SANTO DOMINGO, REPÚBLICA DOMINICANA AL AÑO $2014^{1}$
}

Study of the critical factors for success in the construction of buildings in the city of Santo Domingo, Dominican Republic in 2014

\section{Camila Martínez Samuel Morla Nelson Peña Richard Rosario Carolina Slaiman Heine Solis Damaso Valdez Coral Vargas}

Resumen: En la industria de la construcción se ha estudiado el éxito y cómo obtenerlo, además de las diferentes definiciones se ha llegado a la conclusión de que existen una serie de Factores Críticos que ayudan a alcanzarlo. Para la siguiente investigación, se parte de la lista de Factores Críticos elaborada por Chan, Scott \& Chan (2004), la cual divide en cinco los Factores: que se relacionan con el proyecto, capital humano, y los elementos relacionados con la contratación, el manejo de proyectos externos. A partir

${ }^{1}$ Este artículo contó con la asesoría intelectual del Dr. Tulio Rodríguez. 
del análisis de dichos factores se evalúa y se determina el nivel de importancia y cómo afectan las edificaciones Santo Domingo, República Dominicana. Además se mide la gestión mediante encuestas y contando con tres variables tales como la importancia, ranking y gestión por lo que se tomó una muestra de ingenieros y clientes; como clientes se contemplan aquellos representantes de bienes raíces, compradores en plano y representantes de empresas. Los resultados para los ingenieros es el factor de contrato con una media de 3.82, después está el factor del manejo de proyecto que obtuvo un 3.59, siguiendo con un 2.91 se encuentra el factor humano, luego le sigue los factores relacionados con el proyecto, con un promedio de $2.90 \mathrm{y}$, por último, el factor externo con un 1.84 , todos evaluados en una escala de 5 . Después del proceso de análisis de datos se obtuvo que los factores de mayor importancia son "cumplir con las especificaciones requeridas, el control de calidad" y "estructura de organización".

Palabras clave: Factor crítico, éxito, construcción, edificación, República Dominicana.

Abstract: The construction industry has been studying success and how to get it, besides the different definitions it has been concluded that there are a number of Critical Factors that help us achieve it. The next research starts with a list of Critical Factors produced by Chan, Scott \& Chan which is divided into five factors which are: factors related to the project, human related factors, related to the engagement, project management and external related factors. Knowing these, the factors has been evaluated in Santo Domingo, Dominican Republic and then has been determined those that affect, the level of importance and how they are being managed through surveys and comprising three variables that are importance, ranking and management with a sample of engineers, customers, representatives of real estate and business representatives. The results in order of importance for engineers is the factor of the contract with a 3.82, then the factor of project 
management which earned a 3.59, following a 2.91 is the human factor, then follows the factor of project with an average of 2.90 and finally the external factor with 1.84 all evaluated on a scale of 5. After the process of data analysis it has been obtained that the most important factors are "achieve the specifications and quality control" and "organizational structure".

Keywords: Critical, success, construction, edification, Dominican Republic.

\section{Introducción}

La industria de la construcción es un negocio de mucha competitividad y al mismo tiempo de mucho riesgo por lo tanto hoy en día la construcción de edificaciones es mucho más compleja, lo que provoca que el equipo que participa en dicha industria se enfrente a cambios sin precedentes. En la industria de la construcción se han estudiado los elementos que conducen al éxito y se concluye que para alcanzarlo deben darse tres aspectos fundamentales: tiempo, costo y calidad.

Aun conociendo estos tres parámetros para el éxito es una necesidad identificar los diferentes puntos claves para que estos puedan desarrollarse de modo satisfactorio; llamémoslos factores críticos para el éxito, los cuales están basados en el artículo "Factors Affecting The Success Of A Construction Project", de Albert Chan, David Scott y Ada Chan. Estos investigadores formularon una lista de cinco factores críticos que son los factores relacionados al proyecto, la contratación, la administración del proyecto, factores externos y los factores humanos.

Hay que decir que los factores críticos de éxito de una construcción son numerosos y extensos; en la industria no existe un proceso 
estandarizado a seguir para lograr la correcta gestión de los mismos y por consiguiente el éxito de un proyecto de carácter ingenieril. De este modo, el objetivo de esta investigación es evaluar los factores críticos para el éxito de la construcción de una edificación en la ciudad de Santo Domingo considerando que previamente es preciso determinar los factores críticos que afectan el éxito de un proyecto de esta índole y con esto determinar el nivel de importancia de los factores en la ciudad metropolitana. Finalmente, se busca determinar cómo se están gestionando dichos factores en Santo Domingo con tal de que se logre una guía para darle seguimiento a los puntos principales según el orden de mayor prioridad para alcanzar el anhelado éxito del proyecto.

\section{Antecedentes}

Los términos de factores críticos de éxito los acuña, por primera vez, Ron Daniel. Este autor afirma que los factores críticos de éxito (CSF) son factores o actividades necesarias para asegurar el éxito. Ashley, en 1986, identificó siete factores de éxito y seis criterios de éxito; la diferencia entre factores de éxito y criterios de éxito es que estos últimos son las medidas según las cuales un proyecto es juzgado, mientras que los factores son introducidos al sistema de administración y que redundan en el éxito del proyecto directa o indirectamente (Pakseresht \& Asgari, 2012).

Se han hecho diferentes listas de los factores críticos del éxito. En el artículo más importante en el que se basó la investigación, "Factors Affecting the Success of a Construction Project", los autores apuntan que los factores críticos del éxito pueden agruparse en cinco grandes grupos: factores relacionados con el proyecto, factores relacionados con adquisiciones, factores relacionados con la administración del proyecto, factores relacionados a los participantes y factores externos (Chan, Scott, \& Chan, 2004).

Según Cheong y Mustaffa (2011), los factores críticos para el éxito de un proyecto de construcción pueden agruparse en siete 
macrogrupos que a su vez se dividen en otros subfactores; estos resultados fueron arrojados después de una larga serie de encuestas dirigidas a distintos contratistas, ingenieros y/o empresas. Dichos factores críticos son los siguientes: factores relacionados con el proyecto, factores relacionados con las partes interesadas (cliente), factores relacionados con las partes interesadas (líder de grupo), factores relacionados con las partes interesadas (consultor), factores relacionados con las partes interesadas (contratista), factores de adquisiciones de proyecto y factores externos (Cheong Yong \& Mustaffa, 2011).

Otra lista más reciente de los factores los une en cinco categorías; los factores relacionados con el proyecto, los factores relacionados con los miembros del equipo, los factores relacionados con el cliente, los relacionados con el contratista y los factores relacionados con la administración (Neringa Gudienè, 2013).

\section{3. Éxito en la Construcción}

Un proyecto se define como el logro de un objetivo en específico, el cual envuelve una serie de actividades y tareas que implican consumir recursos. De la misma manera que el artículo "Factors Influencing the Success of Project Management Amongst Manufacturing Companies in Malaysia: a Conceptual Framework" define el criterio como el principio o el estándar por el cual cualquier cosa es o puede ser juzgada (Lim \& Mohamed, 1999).

El éxito, en este mismo tenor, significa diferentes cosas para diferentes personas; cada industria, cada equipo de proyecto y cada persona de manera individual tienen una definición propia del éxito. Incluso la perspectiva de una persona varía de proyecto en proyecto: es por tal razón que el artículo Stakeholders Impact Analysis of Infrastructure Project Management in Developing Countries plantea el éxito como una sensación de perspectiva intangible. El criterio de medición del mismo varía con las expectativas del administrador, entre personas y a través de frases referidas 
a proyectos (Pariff \& Sanvido, 1993). Al ver diferentes definiciones puede concluirse que el concepto de éxito es algo ambiguo pues además de ser complejo por su naturaleza misma, es subjetivo y varía según la perspectiva y el enfoque que cada persona pueda darle. De este modo, la definición de éxito para un proyecto varía de acuerdo con el tamaño, el tipo de proyecto, personal involucrado así como también las experiencias de quienes lo llevan a cabo entre una gran gama de diferentes factores.

Al revisar la literatura referida al tema, el éxito está vinculado a tres importantes factores, o mejor dicho, está medido a través de tres grandes pilares: tiempo, costo monetario y desempeño del proyecto (Chan, 2001). El tiempo, el costo y la calidad de un proyecto han sido las bases sobre las cuales se ha sentado el éxito de un proyecto desde los inicios de investigaciones relacionadas al tema; autores como Walker (1995), Atkinson (1999), Belassi \& Tuckel (1996) y Hatush \& Skitmore (1997) han denominado estos tres pilares como el "Triángulo de Hierro" y aunque definiciones más recientes incluyen otros factores, la definición apoyada en los tres vértices es la más completa y la más utilizada hasta el momento.

Un proyecto que cumpla con: completarse en o por debajo del costo contratado, completarse en o antes de la fecha final del contrato, al momento de finalizar cumple o excede todas las especificaciones técnicas de rendimiento proporcionado por el propietario; cumple o supera los objetivos funcionales previstos del usuario, cumple o excede los estándares aceptados de la mano de obra en todas las áreas y el proceso constructivo del mismo no requiere de la intervención innecesaria del propietario, por lo que es considerado un proyecto exitoso en términos de construcción.

\section{Factores críticos de éxito}

Con los factores críticos para el éxito se busca progresar y asegurar que la industria sea exitosa. Hoy en día la industria de la construcción debe estar preparada para enfrentarse con diferentes 
situaciones entre las cuales pueden mencionarse: manejos de protocolos, manejos de tecnologías y una parte importante que es enfrentarse con la situación financiera. El conocimiento de los factores críticos del éxito crea un puente al mismo tiempo. El estudio de estos factores busca la identificación de las diferentes causas que pueden encaminar un proyecto al éxito y mejorar la efectividad de los mismos en la industria de la construcción. Se busca que las diferentes empresas enfoquen su capacidad en dichos factores para que funcionen de manera más eficiente entendiendo que de esta manera los proyectos se acercan más al éxito (Jari, Pankaj \& Bhangale, 2013). Estos estudios son una manera efectiva de identificar lo esencial para llegar al éxito y se busca que el equipo que trabaja en el proyecto conozca todos los factores que llevan al éxito y se analicen (Yang, Qiping Shen, Drew \& Ho, 2010).

\section{Factores relacionados con el Proyecto}

Dentro de algunas investigaciones y estudios realizados a través del análisis de los factores relacionados con el proyecto de construcción en Bayelsa, Nigeria [se determinó] el efecto que tiene la productividad del trabajo de la construcción. A través de un estudio realizado entre maestros constructores e ingenieros se llegó a la conclusión de que no solo los sub-factores conocidos como: "Los atributos que se utilizan para medir este factor dependen del tipo de proyecto, naturaleza del proyecto, número de pisos del proyecto, complejidad del proyecto y el tamaño del proyecto" (Chan, Scott \& Chan, 2004); no son los únicos para determinar el éxito de un proyecto. Se debe tomar en cuenta también la "especificación y normalización, la disponibilidad del material y objetivos del proyecto e hitos, la alta calidad de las obras necesarias y la disponibilidad de dibujos: son los primeros cinco factores relacionados con los proyectos significativos que afectan la productividad del trabajo de la construcción, respectivamente" (Odesola, Otali \& Ikediashi, 2013: 821). 
La manera correcta para gestionar este factor sería haciendo una revisión de la presentación del proyecto para así observar el nivel de complejidad de este e identificar el tipo de suelo o sector donde este será desarrollado, así como los pisos que se desplegarán. Además habría que revisar la parte administrativa, los factores conocidos como el objetivo del proyecto y la disponibilidad de materiales, los cuales se gestionarían en la presentación del proyecto y en el transcurso de este observar si se está facilitando dicho material para la continuación del desarrollo del proyecto. Las especificaciones y normalizaciones también se analizan antes del comienzo del proyecto y durante el transcurso del mismo. Cuando se presenta una nueva brigada se le debe informar dichas especificaciones antes de empezar a trabajar.

\section{Factores relacionados con la contratación}

La relación que une tanto al contratista como al ingeniero en una obra de construcción es una relación establecida a través de un contrato de trabajo. Dicho contrato debe de contener una idea clara de cómo se manejará no solo el proyecto en sí, sino también lo relativo a las incertidumbres que puedan llegar a ofrecerse, las emergencias, pero sobre todo debe mostrar una idea clara de qué protocolo se llevara a cabo, cuáles serán las sanciones y quién debe ser amonestado a través de la delimitación misma de las actividades entre las partes. Por tanto se determina que el contrato de trabajo es una de las vías más problemáticas al momento de referirnos a las relaciones entre dichas partes (Campero, 2013). La clave para la gestión de dichos factores es entender que los proyectos de construcción son de carácter dinámico, es decir, que los mismos cambian y varían según se gestionan a través de las situaciones que se presentan durante el trayecto de la construcción, pero debe planificarse y comunicarse las decisiones que se toman para las actividades venideras. 
"Por otra parte, existen unos cuantos aspectos a tomar en cuenta, que son los puntos de un contrato que habitualmente generan conflictos y por ende su estudio es de suma importancia, estos son:

a) Definición de la obra contratada, también denominado alcance del contrato.

b) Calidad requerida, que cabe exigir y que se define mediante normas, especificaciones, usos y costumbres.

c) Plazo de ejecución que deberá ser respetado.

d) Precio que importa la contraprestación pactada y que las partes consideran consecuente con los tres aspectos indicados anteriormente" (Bilbao, Campero \& García de la Pastora, 1987: 6).

El factor de contratación es tan importante por el dinero que involucra cualquier error que se presente o cualquier incumplimiento de algunas de sus partes. Es tanto así que en la industria de la construcción anualmente debido a la resolución de disputas en Estados Unidos se gasta de $\$ 4$ a $\$ 12$ billones de dólares (Gebken, 2006) y se ha indicado que en promedio una disputa en esta industria durante el 2011 implicó alrededor de 10.5 millones de dólares y el tiempo que se le dedicó a resolver cada disputa fue de aproximadamente 14.4 meses (Moore \& Riley, 2012).

Un proyecto de construcción tiene las características de que para lograr el resultado esperado no solo basta con hacer cálculos estructurales, de materiales, de mano de obra y construir; sino que hay que enfrentarse en el transcurso del tiempo de la construcción con factores de origen externos al proyecto o la variación del medio (entiéndanse fuerza mayor: Inundación, terremoto, incendio, viento, rayos; Políticos: cambio de leyes y reglamentos, huelgas, permisos y autorizaciones, normas ambientales y de seguridad, embargos; Financiero y económico: inflación, disponibilidad de fondos, fluctuación tipo de cambio) como también enfrentarse con factores de origen interno al proyecto (relacionado con la organización: atraso en permiso para iniciar, atraso de suministros, deficiencias 
de coordinación, deficiencia de control, atraso en soluciones; Relacionados con el diseño: diseño incompleto, defectuoso, errores y omisiones, especificación inadecuada, condiciones de terreno distintas; Relacionado con la construcción: trabajos adicionales, cambios de diseño, condiciones de terreno, trabajo defectuoso, modificación de programas, entre otros). (Campero, 2013)

Al observar todas las variables que pueden presentar un proyecto de construcción es difícil buscar la manera de poder incluir todo en un contrato y aun así poder presentar una licitación competente económicamente hablando, pero se ha demostrado que en muchos casos los malos resultados de los proyectos se debe a que no tomaron en cuenta que la construcción no se desarrolla en un ambiente estático.

Por lo tanto, para la buena gestión de un contrato de construcción es necesario repartir de una manera clara las responsabilidades sobre los efectos previsibles a cada parte así como también contemplar cláusulas de flexibilidad por cualquiera de los factores anteriormente mencionados y así asegurar el cumplimiento de la función del proyecto, con las justas compensaciones en tiempo y costo al contratista para mantener la equidad de la relación contractual (Campero, 2013).

\section{Factores humanos}

El tema de factores los humanos implica tomar el factor como un conjunto de partes que lo forman, se iniciará con el promotor de proyecto o cliente, el constructor, así como lo concerniente al personal obrero. El cliente es la razón de que la industria de la construcción exista, sin embargo, la relación entre el cliente y la industria no siempre es la más favorable (Boyd, 2006). La realidad es que el cliente no se identifica con el proyecto hasta que comienza a ver los espacios construidos y es cuando empiezan las inconformidades e inicia las modificaciones al proyecto, y/o los cambios en especificaciones" (Cervantes Abarca, 2005). En este orden de 
ideas, se evidencia que la relación con el factor cliente estará relacionado con el detalle de lo que se busca en el proyecto y con la comunicación del constructor y el cliente.

"Los directores de proyecto tienen la función de crear conocimiento y compartir los conocimientos e identificar los obstáculos a la adquisición de conocimientos" (Hills, 2008: 6). Esto implica que el director del proyecto o constructor, debe tener las aptitudes necesarias para manejar el personal obrero y comunicar el estado del proyecto al cliente. En la industria de la construcción el personal tiene grandes desventajas por el hecho de que los contratos son limitados a las obras en curso y no son asociados a trabajos fijos, de modo que existe la posibilidad de seguridad (estabilidad económica, activos fijos) o remuneración por antigüedad. La consideración de estos puntos puede representar la forma correcta de gestionar este factor proporcionando cierto grado de seguridad al obrero con la contratación en varias obras (en el caso de tratarse de una constructora); otra forma sería ofrecer capacitación incluso si solo es para un solo proyecto y como consecuencia de esto, remunerar mejor a los obreros más capacitados en distintas áreas.

\section{Factores relacionados al manejo de proyectos}

Los proyectos de construcción son comúnmente criticados por sus retrasos, sus desviaciones de los presupuestos, la baja productividad y su baja calidad (Pilar, Paiva, Dominguez, \& Varajão, 2009). Todos estos problemas están muy apegados a que la gestión de los proyectos, una actividad bastante compleja, conlleva muchas veces un cierto grado de incertidumbre (Varajão, 2014). En la mayoría de los casos, el ciclo de vida del proyecto sigue estas etapas habituales: comienzo; planificación; ejecución/supervisión; y el cierre. La gestión del proyecto es la que se encarga de lo que es la planificación y organización de los recursos con el propósito de cumplir con una meta predeterminada. La administración del proyecto inicia como una necesidad, pero evoluciona a una herramienta para mejorar los procesos constructivos. 
Existen cuatro competencias intrínsecas en el administrador del proyecto que son: la primera se conforma de capacidad técnica, cualidades de liderazgo y actitud positiva; la segunda es delegar responsabilidades a los integrantes del equipo, logrando que los integrantes sientan una relación de pertenencia con la tarea y crea lazos de comunicación entre su equipo; el tercer aspecto es hacerse cargo de las distintas decisiones; y ninguno de estos aspectos ocurriría si el administrador no tomara un rol activo en la construcción (Jha, 2013). Chan et al. (2004) desarrollaron un marco que define a la administración como factor de éxito en los proyectos del ingeniería: "Factores relacionados con la administración del proyecto: sugirieron que mediante el uso de las herramientas de gestión, los administradores de proyectos serían capaces de planificar y ejecutar sus proyectos de construcción para maximizar las posibilidades de éxito del proyecto" (Chan, Scott \& Chan, 2004: 154). El manejo o administración del proyecto logrará el éxito si se monitorean debidamente las actividades previamente coordinadas y programadas.

\section{Factores externos}

Generalmente los investigadores describen la sostenibilidad social como el compromiso de los empleados, las comunidades locales, clientes y la cadena de suministro para asegurar la satisfacción de las necesidades de las poblaciones y las comunidades actuales y futuras (Gambatese, 2008). Burdge presenta una perspectiva que involucra a la comunidad mediante la estimación del impacto de los proyectos de construcción con relación a donde viven los usuarios, donde trabajan, juegan y participan en actividades culturales (Burdge, 2004). Una serie de investigaciones muestran que la sostenibilidad social en un proyecto de construcción se divide en cuatro grupos de gran importancia:

1. La participación de la comunidad enfatiza en las decisiones tomadas por los grupos políticos y privados. 
2. La responsabilidad social que tienen las empresas de prestarle atención a los grupos de interés que son más afectados por las operaciones en sí.

3. Un diseño acerca de mantener la seguridad en lo que ya es el proceso constructivo, es decir hacer un análisis de riesgos del proceso constructivo.

4. Un diseño social centrado en mejorar el proceso de toma de decisiones, si el equipo de diseño y el uso previsto del proyecto por parte de los usuarios finales (Valdés Vásquez, 2013).

Los proyectos públicos son el mayor ejemplo de la presencia gubernamental, en estos casos se requiere de muchos recursos, eficiencia, calidad y transparencia (GFR, 2005). Los proyectos del Estado demandan un alto nivel de transparencia y una buena dirección de cuentas y por lo tanto se le supervisa muy de cerca por auditoría financiera externa y compañías de vigilancias del Estado (Tabish, 2011). Se comprende que los atributos que se utilizan para medir este factor son el entorno económico, el entorno social, el entorno político, el entorno físico, el entorno de relación laboral y el nivel de tecnología avanzada (Chan, Scott, \& Chan, 2004).

En cuanto al ambiente de la localidad del proyecto y al ambiente social, algo que puede ayudar con la seguridad y la tranquilidad de los dueños y de los trabajadores es apelar a la contratación de una compañía de seguridad o simplemente a un guardián. Con respecto al ambiente político y gubernamental, el proceso debería conducirse con la mayor transparencia y calidad dentro de lo posible, en término de las acciones tomadas por la directiva del proyecto. Esto garantizaría la claridad al momento de brindarle un reporte al Estado, de igual modo cuando se realice la supervisión del proyecto, todos los detalles estén en orden. Con relación al ambiente ecológico, el Estado posee reglamentos de todos los procedimientos pro-ambientales que deben tomarse en cuenta en un proyecto y su aplicación correcta. 


\section{Metodología}

Esta investigación pertenece al ámbito correlacional seccional. Correlacional significa que se determinó un número de variables y estas se relacionan entre sí para determinar la lista de factores que definen el éxito en una construcción de edificaciones en la ciudad de Santo Domingo. Y seccional ya que solo se tomarán los datos evaluados en la actualidad.

Se tendrán variables e indicadores para levantar información. Lo primero que se investigó fue la presencia: esta variable hace referencia a determinar si los factores planteados se encuentran dentro de las obras de edificaciones de la ciudad de Santo Domingo, es decir, si los mismos tienen aplicación dentro del campo de nuestra investigación. Luego se evalúa la importancia de cada factor, dicha variable indica entre los factores seleccionados cual influye más en el éxito de la construcción de edificaciones. Por último, se elaborará un ranking de factores con el objetivo de ordenar los mismos atendiendo al nivel de importancia impuesto por los encuestados.

Estos datos se determinaron mediante las encuestas realizadas a noventa (90) ingenieros encargados de obras de edificación en la ciudad de Santo Domingo; se les pidió que organizaran del 1 al 5 los cinco factores generales obtenidos en la investigación teórica de acuerdo con su opinión, siendo 1 el que menos afecta y 5 el que más afecta el éxito en la construcción de edificaciones.

Luego se realiza una comparación entre los ingenieros y los clientes. $\mathrm{Al}$ hacer referencia a los clientes, cabe destacar tres grandes grupos: clientes generales (personas que adquieren viviendas en construcción) (35 personas), clientes de bienes raíces (personas que venden edificaciones) (13 personas) y clientes de empresas (empresas con crecimiento de infraestructura) (7 empresas). Esta comparación será compilada por medio de la misma encuesta realizada a los ingenieros, sin tomar en cuenta las preguntas más técnicas, para mayor entendimiento de la misma. 
Los datos recolectados a través de las encuestas, luego de ser procesados y depurados con la finalidad de que los mismos no hayan sido alterados y/o manipulados dentro del proceso de recolección fueron analizados a través de algoritmos estadísticos. El software a implementarse para la interpretación de los datos fue SPSS (Statistical Package for the Social Sciences o Statistical Products and Services Solutions), el cual es un programa estadístico informativo que se implementa con la finalidad de analizar de forma estadística los datos recolectados durante el proyecto para poder generar conclusiones y contribuir con la interpretación de la investigación.

\section{Presentación de los resultados}

Con los resultados obtenidos mediante la encuesta se determinó el orden o ranking de los cinco factores generales tal como se muestra en la figura $\mathrm{N} .^{\circ} 1$.

Figura N. ${ }^{\circ} 1$

Ranking de factores

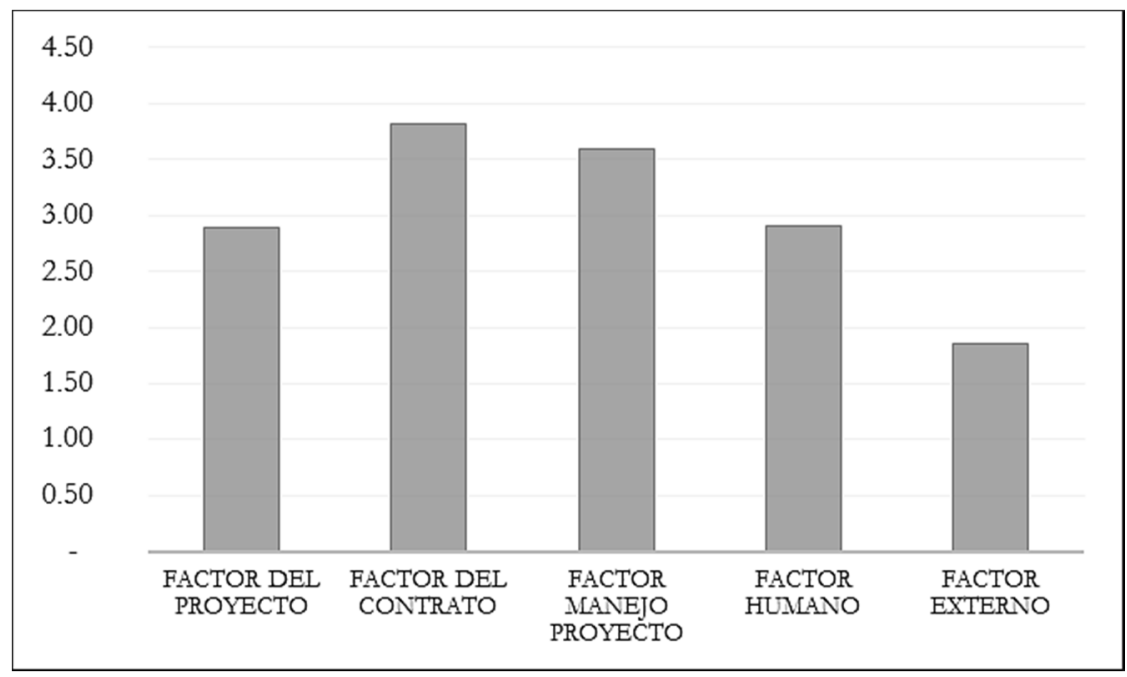


Los resultados arrojados muestran cómo el factor contrato resulta ser el factor general más crítico para los ingenieros, con una media de 3.82. A este le sigue el factor manejo del proyecto con una media de 3.59; el factor humano con una media de 2.91; el factor relacionado con proyecto con una media de $2.90 \mathrm{y}$, por último, considerado para los ingenieros como el factor que menos afecta el éxito se encuentra el factor externo con una media de 1.84; dichas medidas están escaladas en base a 5 .

Cada factor general anteriormente presentado poseía dentro de sí algunos sub-factores que también fueron evaluados en particular. En esta parte se les pidió a los encuestados valorar cada uno de los sub-factores tomando en cuenta la siguiente escala: del 1 al 4, siendo 1 = Irrelevante, 2 = Parcialmente importante, $3=$ Importante, 4=Imprescindible, para el éxito de una construcción de edificaciones en la ciudad de Santo Domingo. A continuación se presentan los resultados.

En la siguiente tabla puede apreciarse no solo los resultados de los promedios otorgados por las encuestas sino también a qué factor o grupo de sub-factores pertenece. Al observar los resultados, se evidencia que los sub-factores de mayor incidencia son especificaciones de calidad y planificación acordados para el proyecto; con estos resultados podemos apreciar con mayor claridad que la mayoría de los factores encuestados representan un aspecto importante para la construcción; sin embargo, algunos resultan irrelevantes como se aprecia en su mayoría en los factores humanos. Lo mostrado en la tabla puede producir cierto desconcierto, ya que en otros países donde se han realizado este tipo de investigación uno de los factores más importantes es el humano. La razón de ser de esto, a nuestro juicio, radica en que la cultura del país es propensa a desfavorecer a la clase trabajadora, por tanto no se evalúa el rendimiento ni la productividad de los obreros cuando están en situaciones de confort. Las evaluaciones realizadas más adelante desglosan con mayor detalle estos resultados obtenidos. 
Estudio de los factores críticos para el éxito en la construcción de edificaciones en la ciudad de Santo Domingo, República Dominicana al año 2014

Tabla N. ${ }^{\circ} 1$

Ranking de importancia de sub-factores para el éxito

\begin{tabular}{|c|l|c|c|}
\hline Ranking & \multicolumn{1}{|c|}{ Sub-factor para el éxito } & Media & $\begin{array}{c}\text { Factor } \\
\text { General }\end{array}$ \\
\hline $\mathbf{1}$ & Especificación de calidad & 3.7 & FRC \\
\hline $\mathbf{2}$ & Planificación acordada para el proyecto & 3.5 & FMP \\
\hline $\mathbf{3}$ & Tiempo de entrega establecido & 3.5 & FRC \\
\hline $\mathbf{4}$ & Cumplimiento de la planificación & 3.4 & FMP \\
\hline $\mathbf{5}$ & Cumplimiento de los códigos del estado & 3.4 & FE \\
\hline $\mathbf{6}$ & Presupuesto acordado & 3.3 & FRC \\
\hline $\mathbf{7}$ & $\begin{array}{l}\text { Buena comunicación entre las partes involucradas } \\
\text { del proyecto }\end{array}$ & 3.3 & FMP \\
\hline $\mathbf{8}$ & Cumplimento de los códigos de medio ambiente & 3.3 & FE \\
\hline $\mathbf{9}$ & $\begin{array}{l}\text { Habilidad del supervisor de delegar } \\
\text { responsabilidades }\end{array}$ & 3.3 & FH \\
\hline $\mathbf{1 0}$ & Características específicas del entorno & 3.3 & FRP \\
\hline $\mathbf{1 1}$ & Obreros trabajando de forma segura & 3.2 & FH \\
\hline $\mathbf{1 2}$ & $\begin{array}{l}\text { Conocimiento del supervisor de los roles de los } \\
\text { involucrados en el proyecto }\end{array}$ & 3.2 & FH \\
\hline $\mathbf{1 3}$ & Preparación del obrero & 3.1 & FH \\
\hline $\mathbf{1 4}$ & Agilidad del cliente para tomar decisiones & 3 & FH \\
\hline $\mathbf{1 5}$ & $\begin{array}{l}\text { Habilidad del supervisor para transmitir un } \\
\text { ambiente de confort para el trabajo }\end{array}$ & 3 & FH \\
\hline $\mathbf{1 6}$ & Gran dificultad de los procesos constructivos & 2.9 & FRP \\
\hline $\mathbf{1 7}$ & $\begin{array}{l}\text { Capacidad del supervisor para tomar circunstancias } \\
\text { como oportunidades }\end{array}$ & 2.9 & FH \\
\hline $\mathbf{1 8}$ & Decisiones gubernamentales variables & 2.8 & FE \\
\hline $\mathbf{1 9}$ & Participación del cliente en el proceso constructivo & 2.7 & FH \\
\hline $\mathbf{2 0}$ & Ubicación del almacén & 2.4 & FMP \\
\hline $\mathbf{2 1}$ & Nivel de relaciones sociales en el entorno de trabajo & 1.8 & FE \\
\hline $\mathbf{L e y}$ & & \\
\hline
\end{tabular}

\section{Leyenda:}

FRP $=$ Factores Relacionados con el Proyecto

FRC $=$ Factores Relacionados con el Contrato

FMP $=$ Factores del Manejo del Proyecto

$\mathrm{FH}=$ Factores Humanos

$\mathrm{FE}=$ Factores Externos 


\section{Evaluación en SPSS para comparaciones múltiples}

Al obtener el orden de incidencia que según los ingenieros tenía cada factor en la construcción de edificaciones en la ciudad de Santo Domingo se encontró que algunas características de los ingenieros estaban íntimamente relacionadas con el algún factor y los factores que se relacionaban entre sí. Se introdujeron los datos en el programa estadístico SPSS con tal evaluar si entre los datos demográficos de los ingenieros y los factores había alguna relación y/o tendencia, además de evaluar si dentro de los mismos factores había una relación directamente proporcional.

\subsection{Primer caso}

En la tabla siguiente se muestra la relación entre el costo del proyecto con tal de cumplir con el tiempo de entrega establecido, se observa cómo el Chi-cuadrado de Pearson da 0.009 esto indica una diferencia estadísticamente significativa, es decir, a mayor costo del proyecto más importante es la variable "cumplir con el tiempo de entrega establecido". En la primera tabla se observa cómo dependiendo del rango del costo del proyecto, la cantidad de encuestados que seleccionaron cada categoría de importancia. Mientras que en la segunda tabla se presenta la prueba estadística de Pearson.

Tabla N. ${ }^{\circ} 2$

Recuento - Primer caso

\begin{tabular}{|c|c|c|c|c|}
\hline \multirow{2}{*}{$\begin{array}{l}\text { Costo del } \\
\text { proyecto }\end{array}$} & \multicolumn{3}{|c|}{ Cumplir con el tiempo de entrega establecido } & \multirow[b]{2}{*}{ Total } \\
\hline & $\begin{array}{c}\text { Parcialmente } \\
\text { Importante } \\
\end{array}$ & Importante & Imprescindible & \\
\hline $0-10 \mathrm{MM}$ & 1 & 1 & 4 & 6 \\
\hline $10 \mathrm{MM}-40 \mathrm{MM}$ & 1 & 4 & 15 & 20 \\
\hline $40 \mathrm{MM}-150 \mathrm{MM}$ & 3 & 15 & 6 & 24 \\
\hline $150 \mathrm{MM}-\mathrm{mas}$ & 0 & 18 & 22 & 40 \\
\hline Total & 5 & 38 & 47 & 90 \\
\hline
\end{tabular}


Estudio de los factores críticos para el éxito en la construcción de edificaciones en la ciudad de Santo Domingo, República Dominicana al año 2014

Tabla N. ${ }^{\circ} 3$

Pruebas de chi-cuadrado - Primer caso

\begin{tabular}{|l|c|c|c|}
\hline & Valor & gl & $\begin{array}{c}\text { Sig. asintótica } \\
\text { (bilateral) }\end{array}$ \\
\hline Chi-cuadrado de Pearson & $17.019(\mathrm{a})$ & 6 & .009 \\
Razón de verosimilitudes & 19.329 & 6 & .004 \\
Asociación lineal por lineal & .099 & 1 & .753 \\
N de casos válidos & 90 & & \\
\hline
\end{tabular}

(a) 6 casillas $(50.0 \%)$ tienen una frecuencia esperada inferior a 5. La frecuencia mínima esperada es 0.33

\subsection{Segundo caso}

En la tabla siguiente se muestra la relación entre el costo del proyecto con obreros trabajando de forma segura y confortable, se observa cómo el Chi-cuadrado de Pearson da 0.044, lo que indica una diferencia estadísticamente significativa, es decir, mientras mayor es el costo del proyecto el factor Obreros trabajando de forma segura y confortable adquiere mayor importancia. En la primera tabla se observa cómo mientras aumenta el rango del costo del proyecto, la cantidad de encuestados que opinan que este factor es importante e imprescindible también aumenta. Mientras que en la segunda tabla se presenta la prueba estadística de Pearson.

\section{Tabla N. ${ }^{\circ} 4$}

Recuento - Segundo caso

\begin{tabular}{|c|c|c|c|c|c|}
\hline \multirow{2}{*}{$\begin{array}{c}\text { Costo del } \\
\text { proyecto }\end{array}$} & \multicolumn{3}{|c|}{ Obreros trabajando de forma segura y confortable } & \multirow{2}{*}{ Total } \\
\cline { 2 - 5 } & Irrelevante & $\begin{array}{c}\text { Parcialmente } \\
\text { Importante }\end{array}$ & Importante & Imprescindible & \\
\hline $0-10 \mathrm{MM}$ & 0 & 0 & 6 & 0 & 6 \\
$10 \mathrm{MM}-40 \mathrm{MM}$ & 0 & 1 & 9 & 10 & 20 \\
$40 \mathrm{MM}-150 \mathrm{MM}$ & 0 & 4 & 9 & 11 & 24 \\
$150 \mathrm{MM}-\mathrm{mas}$ & 3 & 5 & 10 & 22 & 40 \\
\hline Total & $\mathbf{3}$ & $\mathbf{1 0}$ & $\mathbf{3 4}$ & $\mathbf{4 3}$ & $\mathbf{9 0}$ \\
\hline
\end{tabular}


Tabla N. ${ }^{\circ} 5$

Pruebas de chi-cuadrado - Segundo caso

\begin{tabular}{|l|c|c|c|}
\hline & Valor & gl & $\begin{array}{c}\text { Sig. asintótica } \\
\text { (bilateral) }\end{array}$ \\
\hline Chi-cuadrado de Pearson & $17.322(\mathrm{a})$ & 9 & .044 \\
Razón de verosimilitudes & 20.320 & 9 & .016 \\
Asociación lineal por lineal & .003 & 1 & .956 \\
N de casos válidos & 90 & & \\
\hline
\end{tabular}

(a) Diez casillas $(62.5 \%)$ tienen una frecuencia esperada inferior a 5. La frecuencia mínima esperada es 0.20

\subsection{Tercer caso}

Se realizaron pruebas estadísticas para determinar cómo afectan las variables de experiencia del ingeniero y el costo del proyecto a los distintos grupos de factores (Factores relacionados con el Proyecto, con el Contrato, con lo relativo al Manejo de Proyecto, factores humanos y factores externos. Sin embargo, se determinó que solo existe una diferencia significativa en la comparación de la relación experiencia del ingeniero y los factores relacionados con el Proyecto.

A continuación se presenta la comparación de los años de experiencia de los ingenieros encuestados y el promedio de los factores relacionados con el Proyecto. En la primera tabla se observa la prueba Anova mediante la cual se determinó que hay una diferencia estadísticamente significativa de 0.044 , lo que indica que mientras más experiencia tengan los ingenieros, más importancia se les otorga a los factores relacionados con el proyecto. Al observar la segunda tabla, es decir, la tabla de comparaciones múltiples, se determina que la progresión de los encuestados es que a mayor experiencia laboral más importancia le dan a los factores relacionados con el proyecto. 
Estudio de los factores críticos para el éxito en la construcción de edificaciones en la ciudad de Santo Domingo, República Dominicana al año 2014

\section{Anova}

Tabla N. ${ }^{\circ} 6$

Factores relacionados con el proyecto

\begin{tabular}{|c|c|c|c|c|c|}
\hline & $\begin{array}{c}\text { Suma de } \\
\text { cuadrados }\end{array}$ & g1 & $\begin{array}{c}\text { Media } \\
\text { cuadrática }\end{array}$ & F & Sig. \\
\hline Inter-grupos & 3.698 & 3 & 1.233 & 2.820 & .044 \\
Intra-grupos & 37.591 & 86 & .437 & & \\
Total & 41.289 & 89 & & & \\
\hline
\end{tabular}

Tabla N. ${ }^{\circ} 7$

Comparaciones múltiples

Variable dependiente: Factores relacionados con el proyecto - DMS

\begin{tabular}{|c|c|c|c|c|c|c|}
\hline \multirow{2}{*}{$\begin{array}{l}\text { (I) Años de } \\
\text { Experiencia }\end{array}$} & \multirow{2}{*}{$\begin{array}{l}\text { (J) Años de } \\
\text { Experiencia }\end{array}$} & \multirow{2}{*}{$\begin{array}{l}\text { Diferencia de } \\
\text { medias (I-J) }\end{array}$} & \multirow{2}{*}{$\begin{array}{l}\text { Error } \\
\text { típico }\end{array}$} & \multirow{2}{*}{ Sig. } & \multicolumn{2}{|c|}{$\begin{array}{c}\text { Intervalo de con- } \\
\text { fianza al } 95 \%\end{array}$} \\
\hline & & & & & $\begin{array}{l}\text { Límite } \\
\text { superior }\end{array}$ & $\begin{array}{l}\text { Límite } \\
\text { inferior }\end{array}$ \\
\hline \multirow{3}{*}{ 0-10 años } & $11-20$ años & -.03788 & .21012 & .857 & -.4556 & .3798 \\
\hline & 21-30 años & .35606 & .18197 & .054 & -.0057 & .7178 \\
\hline & $\begin{array}{l}\text { más de } 30 \\
\text { años }\end{array}$ & $.44251(*)$ & .21349 & .041 & .0181 & .8669 \\
\hline \multirow{3}{*}{$11-20$ años } & 0-10 años & .03788 & .21012 & .857 & -.3798 & .4556 \\
\hline & 21-30 años & $.39394(*)$ & .19372 & .045 & .0088 & .7790 \\
\hline & $\begin{array}{l}\text { más de } 30 \\
\text { años }\end{array}$ & $.48039\left(^{*}\right)$ & .22360 & .034 & .0359 & .9249 \\
\hline \multirow{3}{*}{$21-30$ años } & 0-10 años & -.35606 & .18197 & .054 & -.7178 & .0057 \\
\hline & $11-20$ años & $-.39394\left(^{*}\right)$ & .19372 & .045 & -.7790 & -.0088 \\
\hline & $\begin{array}{l}\text { más de } 30 \\
\text { años }\end{array}$ & .08645 & .19738 & .662 & -.3059 & .4788 \\
\hline \multirow{3}{*}{$\begin{array}{l}\text { Más de } 30 \\
\text { años }\end{array}$} & 0-10 años & $-.44251\left(^{*}\right)$ & .21349 & .041 & -.8669 & -.0181 \\
\hline & $11-20$ años & $-.48039(*)$ & .22360 & .034 & -.9249 & -.0359 \\
\hline & 21-30 años & -.08645 & .19738 & .662 & -.4788 & .3059 \\
\hline
\end{tabular}

* La diferencia de medias es significativa al nivel 0.05 
Al conocer el nivel de significancia estadística, podemos concluir que existe una diferencia estadísticamente significativa, es decir, al relacionar la variable dependiente de los factores relacionados con el proyecto y este con los años de Experiencia por lo que se determina que los años de Experiencia afecta al mismo.

\section{Gestión de factores}

Al encontrar los factores críticos se aspiró a investigar la manera en que se estaban gestionando los dos sub-factores más críticos para el éxito en la construcción de edificaciones de Santo Domingo. Para esto, se visitó a 27 obras en las que se examinó si poseían o no una política de calidad y una planificación.

\subsection{Cumplir con las especificaciones de calidad requerida}

Tabla N. ${ }^{\circ} 8$

Obras que poseen una política de calidad

\begin{tabular}{|l|c|c|c|}
\hline & Buena & Débil & No existe \\
\hline Cantidad & 17 & 5 & 5 \\
\hline Porcentaje & $63 \%$ & $19 \%$ & $19 \%$ \\
\hline
\end{tabular}

Al realizar el estudio de campo en diferentes construcciones de edificaciones en la ciudad de Santo Domingo se determinó que alrededor de un $82 \%$ de la muestra tomada posee una política de calidad en su proceso constructivo y un $63 \%$ de estos afirma que dicha política es buena.

Para los encuestados su política de calidad consistía en:

- Control y chequeo en cada una de las áreas en la construcción, específicamente, en materiales, en lo concerniente a la colocación de ellos y la supervisión de la obra en general. 
- Verificación de propiedades y características físicas del terreno, control de la separación de acero, verificación de las propiedades del hormigón y supervisar el encofrado, la madera, los puntales, los clavos y la dosificación de pañete.

- Verificar si los materiales más costosos cumplían con las especificaciones requeridas, hacer pruebas de probetas, etc.

- Verificación de propiedades del hormigón y cumplir con el plano, entre otras.

Luego de examinar dichas respuestas se podría concluir que el sector de la construcción de edificaciones posee buenas políticas de calidad para llevar a cabo todas las especificaciones de calidad requeridas por el cliente, pero al compararla con la forma de hacer una correcta gestión se ve que no es así. Las visitas al campo demostraron que en una gran parte de las obras se consideraba como una buena política de calidad, el revisar cosas básicas como: hormigón, terreno y medidas; y lamentablemente solo una minoría tenían esta política de forma escrita. Esto llega a preocupar en gran manera, ya que si el sub-factor "Cumplir con las especificaciones de calidad requeridas" es el sub-factor que más afecta la construcción, velar por su cumplimiento debería ser de una manera más detallada y minuciosa.

\section{Velar por el cumplimiento de la planificación durante el proyecto obras que poseen una planificación}

Tabla N. ${ }^{\circ} 9$

Obras que poseen una política de calidad

\begin{tabular}{|c|c|c|}
\hline & Poseen & No poseen \\
\hline Cantidad & 18 & 9 \\
\hline Porcentaje & $67 \%$ & $33 \%$ \\
\hline
\end{tabular}


Para estudiar el segundo factor más importante para el éxito de una construcción se realizaron visitas a distintas obras con el fin de encontrar cómo se estaba gestionando dicho sub-factor. Se descubrió que un $67 \%$ de las obras poseen o afirman poseer una planificación para organizar las actividades que realizarán en el día y en la semana.

La mayoría de los encuestados aseguran tener dicha planificación realizadas en diagramas de Gantt u otros tipos de cronogramas, por otra parte, algunos expresaban que se realizaba una reunión semanal para programar los pasos a seguir en la semana según se veía la necesidad; otros mencionaron que diariamente se les decía que se realizaría ese día y otros por flujo de brigada. Estas planificaciones en su mayoría eran supervisadas por los ingenieros o arquitectos residentes o maestros constructores.

De igual modo, al comparar la planificación con la correcta gestión se descubre que este factor se encuentra débil en Santo Domingo, ya que aunque se halla de manera más habitual en las construcciones no se está realizando y gestionando de modo adecuado, porque las planificaciones no se realizan como deberían ni se llevan a cabo. Estas no se realizan de la manera correcta debido a que aunque en la mayoría de las obras se elaboran listados de las actividades que se llevarán a cabo, tampoco se especifica qué duración tendrá cada una de estas. Esto provoca que no se tenga un control de lo que debe ser realizado en ese día y en qué tiempo debe ser realizado y como consecuencia ocurren retrasos innecesarios en las obras ingenieriles como son retrasos en los pedidos, no poder continuar con un trabajo debido a que es necesario que finalice otro para comenzar, entre otros. Si bien es cierto que dentro de los analizados existían planificaciones bien elaboradas (dígase especificando el tiempo necesario), no lo es menos que muchas no toman dichas planificaciones y van trabajando sobre la marcha y esperan el momento exacto para realizar la actividad sin planificar con anterioridad. Esto también se traduce en retrasos en las obras de construcción. 


\section{Resultados obtenidos encuestas clientes}

Se realizó una comparación entre lo que consideran los ingenieros que es el éxito con relación a lo que opinan los clientes. Al hacer referencia a los clientes, cabe destacar tres grandes grupos:

- Clientes generales,

- Clientes de bienes raíces

- Clientes de empresas

Cliente general: Son aquellas personas que adquieren o realizan una transacción por la compra de una edificación en construcción al momento o antes de dar inicio a la construcción, los que comúnmente denominamos como compradores en plano.

Clientes bienes raíces: Son personas que se dedican a comercializar y prestar servicios de mercadeo para gestionar la venta de una edificación, es decir, aquellos intermediarios entre el cliente (comprador) y el constructor y/o dueño del inmueble.

Cliente de empresas: Son aquellos departamentos encargados de clasificar y contratar empresas constructoras para la elaboración de edificaciones a construirse por la empresa así como también velar para que los mismos presten un servicio adecuado a las necesidades y especificaciones esperadas por la empresa.

En la gráfica que se muestra a continuación, se aprecian los promedios de los valores obtenidos al puntuar los diferentes factores que juegan distintos papeles dentro de las obras ingenieriles y sus comparaciones con los distintos tipos de cliente. 


\section{Figura N. ${ }^{\circ} 2$}

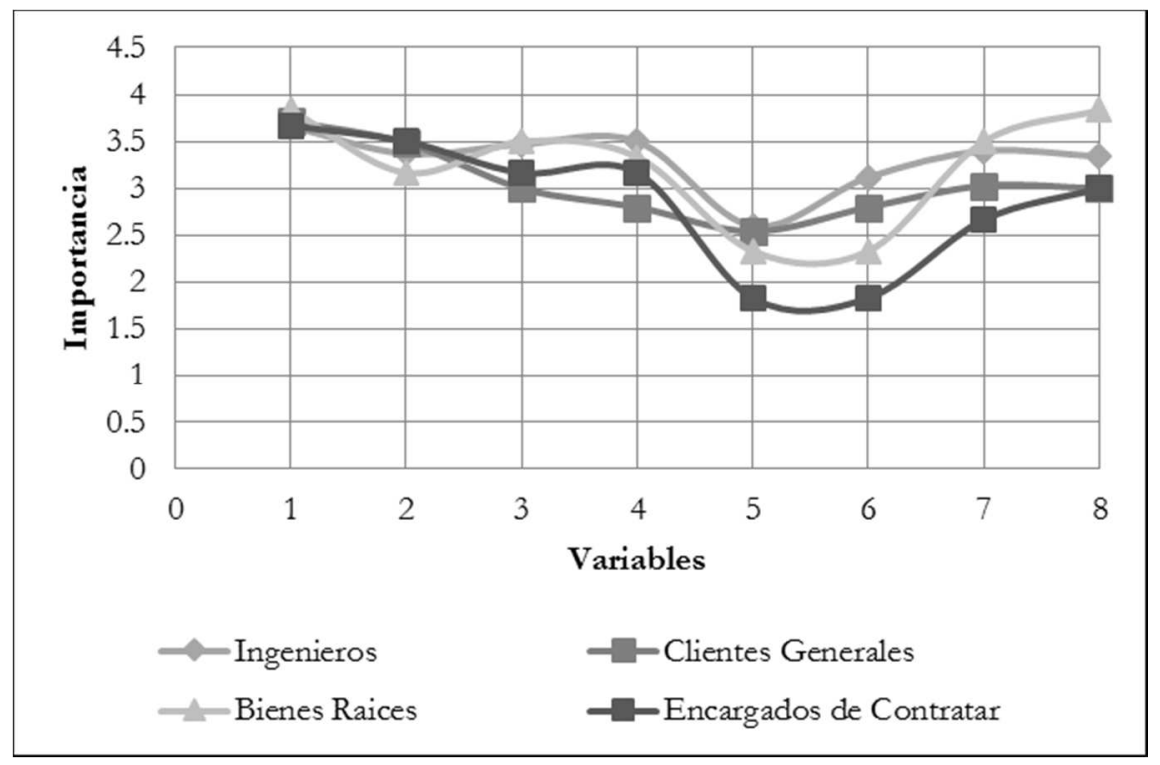

\section{Leyenda:}

\begin{tabular}{|l|l|}
\hline N. $^{\circ}$ & Variables \\
\hline 1 & Especificaciones de calidad requeridas \\
\hline 2 & Presupuesto acordado \\
\hline 3 & Tiempo de entrega establecido \\
\hline 4 & Planificación acorde al proyecto \\
\hline 5 & Participación del cliente en el proceso constructivo \\
\hline 6 & Agilidad del cliente de tomar decisiones \\
\hline 7 & Cumplimiento de los códigos de Estado \\
\hline 8 & Cumplimiento de los códigos de Medio Ambiente \\
\hline
\end{tabular}

Se evaluó cada factor con una prueba Anova y los resultados obtenidos fueron los siguientes: 
Caso 1: Factor que involucra el cumplimiento de las especificaciones de calidad requeridas

\section{Figura N. ${ }^{\circ} 3$ - Caso 1}

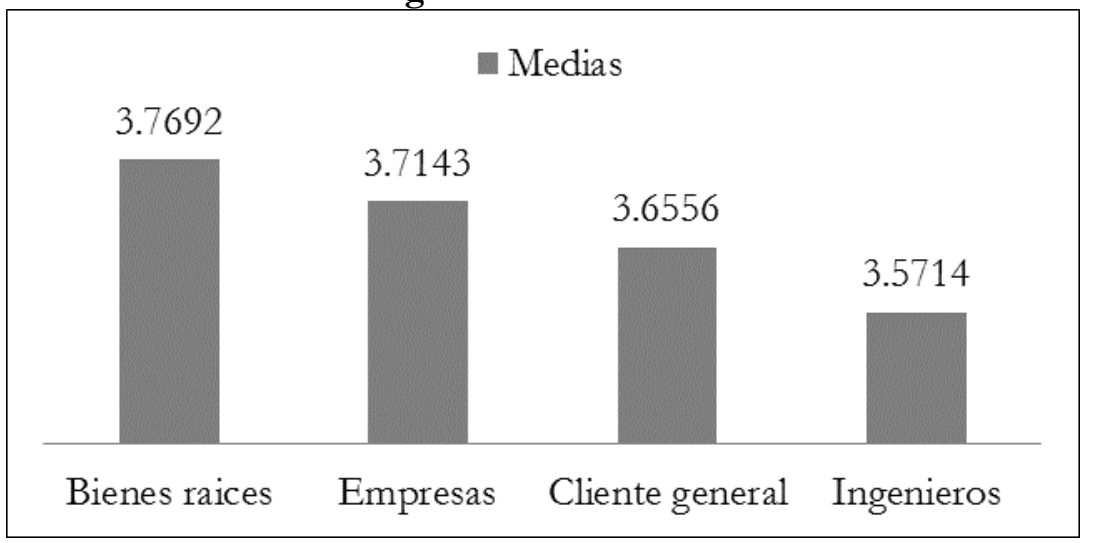

El nivel de significancia es de 0.825 por lo que no hay una diferencia de las opiniones. Cumplir con las especificaciones de calidad requeridas es el factor más importante tanto para los ingenieros como para los clientes atendiendo a las medias observadas con respecto a los demás factores.

\section{Caso 2: Factor cumplir con el presupuesto acordado}

Figura N. ${ }^{\circ} 4$ - Caso 2

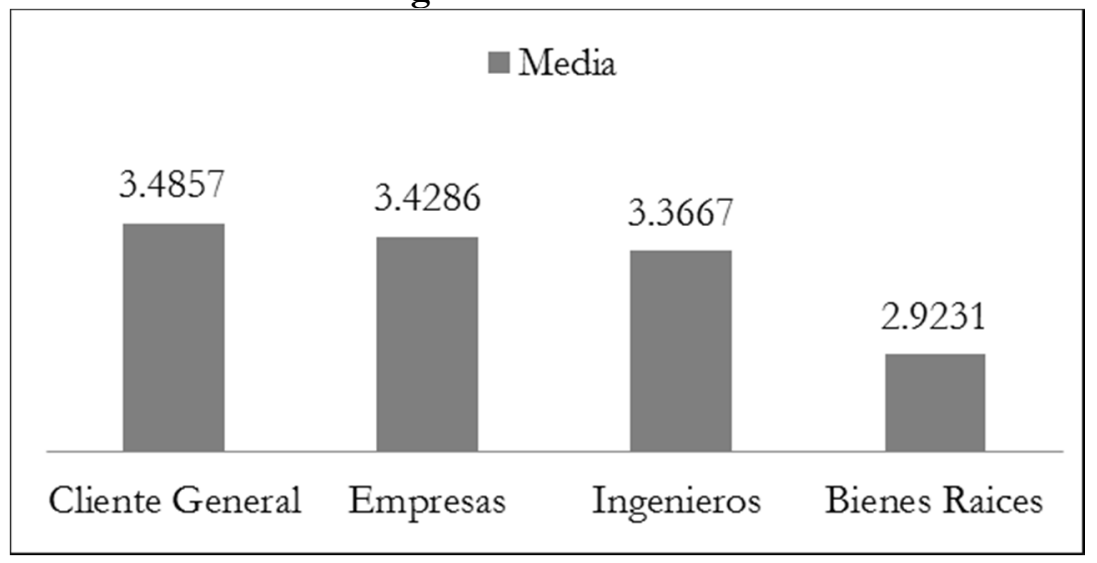


Dentro de la gráfica, se muestra que la media más baja pertenece al gremio de los clientes de bienes raíces, esto se debe a que las variaciones del presupuesto luego de ser firmado el contrato son responsabilidad del ingeniero o del cliente, sin embargo, el agente le da un grado de importancia ya que mientras exista una variación en el contrato puede tener una repercusión en un cliente insatisfecho que resulta en una mala publicidad para el mismo. El nivel de significancia en la prueba Anova es de 0.039, lo que significa que hay una diferencia significativa, es decir, los clientes bienes raíces difieren de todos los demás encuestados.

\section{Caso 3: Factor cumplir con el tiempo de entrega establecido}

Figura N. ${ }^{\circ}$ - Caso 3

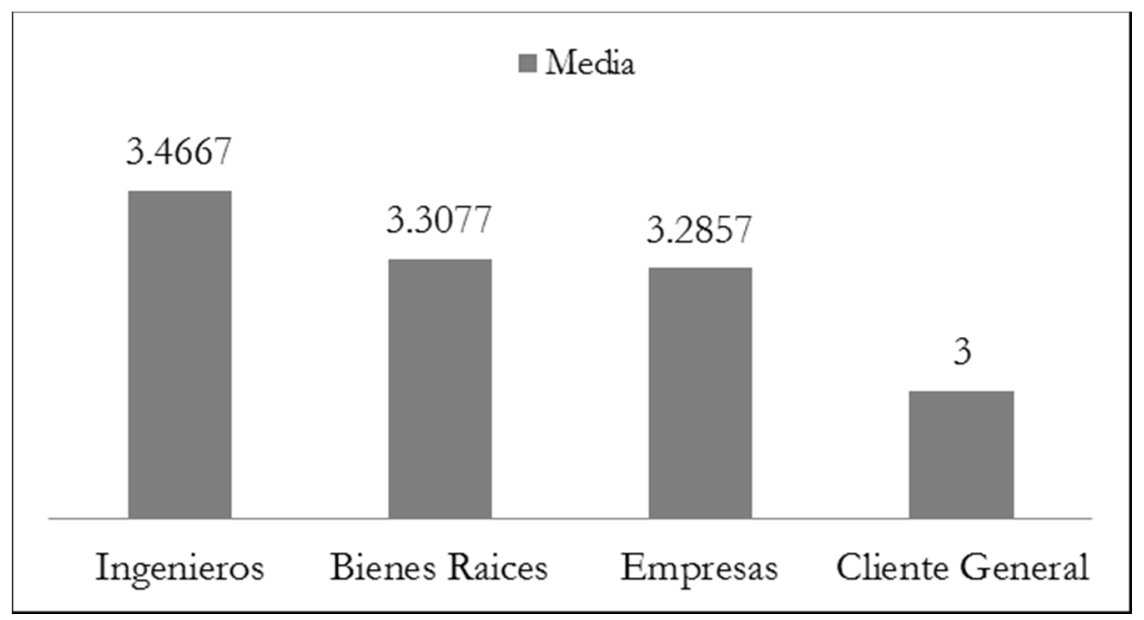

Puede verse que el tipo de encuestado es el que considera más importante este factor contra los demás y que el cliente general es el que lo considera menos importante, esto ocurre ya que los residentes de Santo Domingo tienen la cultura de esperar que las construcciones se atrasen, la mayoría de personas no compra en 
plano si tiene que ocupar la vivienda en un tiempo específico. El nivel de significancia estadística de 0.006 muestra que existe una diferencia estadísticamente significativa.

\section{Caso 4: Factor velar por el cumplimiento de la planificación durante el proyecto}

Figura N. ${ }^{\circ} 6-$ Caso 4

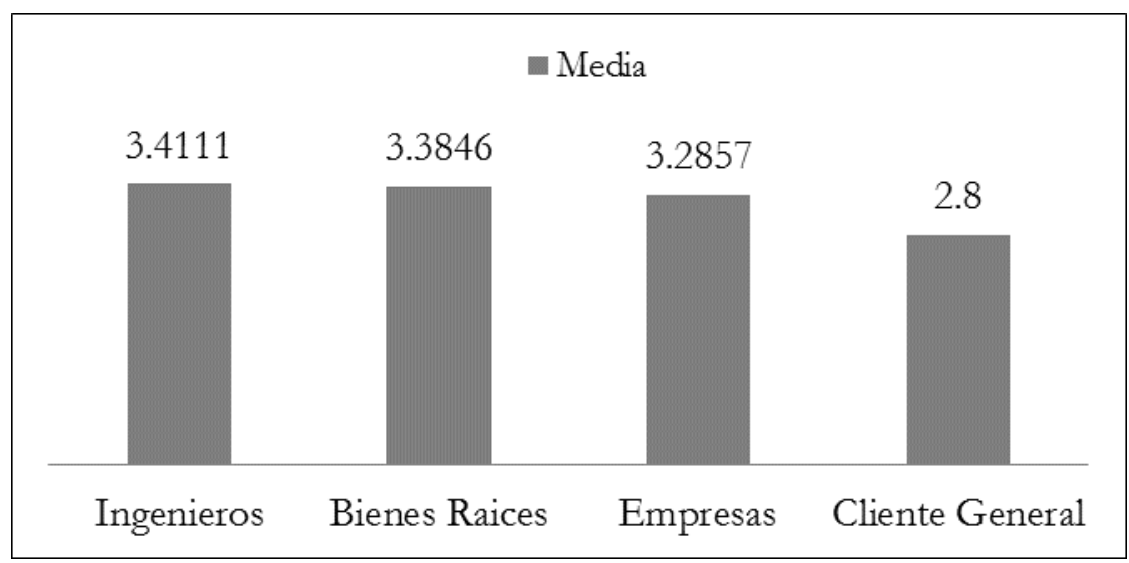

Puede observarse cómo el único tipo de cliente que difiere en el nivel de importancia es el cliente general, esto se debe a que el factor evaluado es parte fundamental del proceso constructivo, proceso en el cual el cliente general no está ligado en la gran mayoría de los casos, por lo que no resulta tan importante velar por la planificación. Y desde el otro punto de vista, están los ingenieros, empresas y bienes raíces que deben preocuparse porque dicha planificación se cumpla ya que es la herramienta de evaluar y pronosticar el desarrollo de la obra. El nivel de significancia es de 0.000, se nota que existe una diferencia estadísticamente significativa entre las opiniones de los encuestados. 


\section{Caso 5: Factor participación del cliente en el proceso constructivo}

Figura N. ${ }^{\circ} 7$ - Caso 5

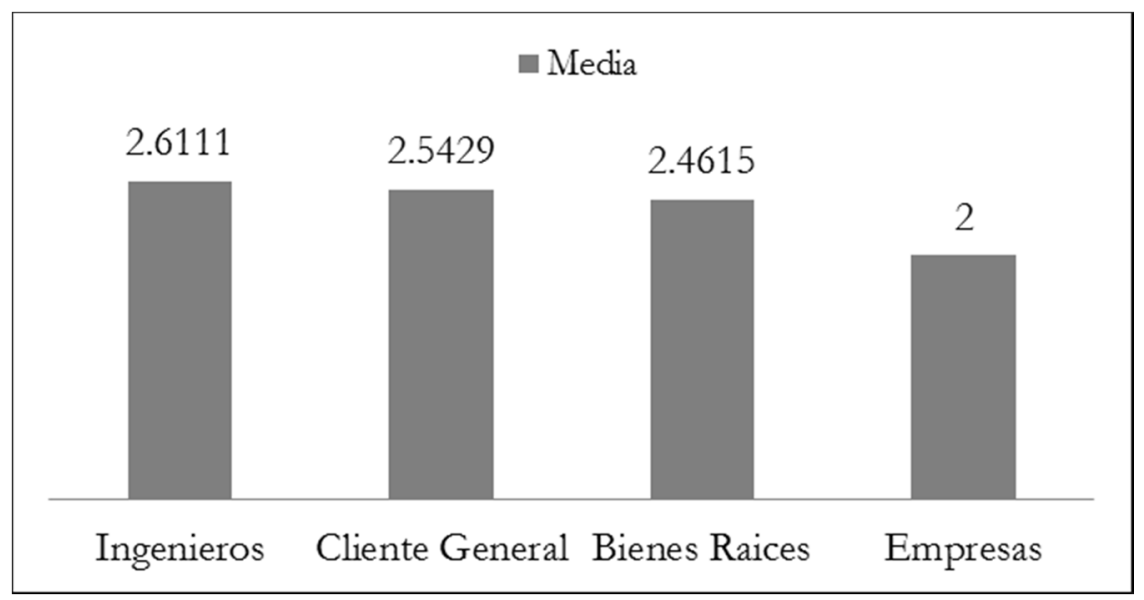

El nivel de significancia es de 0.399 por lo que no existe una diferencia significativa, sin embargo cabe destacar que los resultados no coinciden con la literatura estudiada anteriormente. Por ejemplo al leer el artículo "Por qué una empresa debería tomar el control de sus proyectos de edificación”, se observa que gracias a las modificaciones en el diseño cuando la empresa participó en el proceso constructivo pudieron lograrse las reducciones en los costos. Además de mostrar la imagen que ellos querían lograr de transparencia, es decir, gracias a que el cliente participó en el proceso de construcción fue un éxito y logró la satisfacción del cliente en su máxima expresión (Thrum, 2005). 


\section{Caso 6: Factor alta capacidad y agilidad del cliente para tomar decisiones por cada cliente}

Figura N. ${ }^{\circ} 8-$ Caso 6

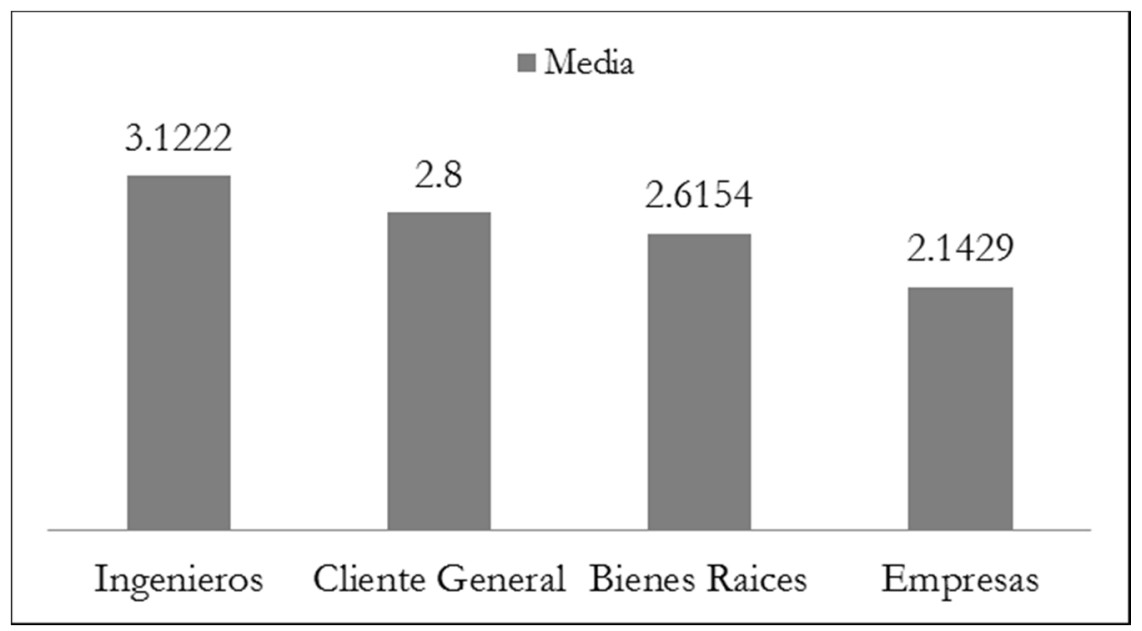

Puede apreciarse que para las empresas es parcialmente importante, a diferencia, del resto de los encuestados. Esto es debido a que como en los contratos y demás documentos, las empresas plasman y detallan todas las especificaciones y requerimientos que desean que posea la obra por la cual están pagando, además en el contrato ya pueden venir establecidos los tiempos de respuestas máximos del cliente.

\section{Conclusiones}

En resumen, después de varias investigaciones se llegó a la conclusión de que en República Dominicana se encuentran cinco (5) grandes factores críticos que afectan el éxito en un proyecto de construcción que son los siguientes: factores relacionados con el proyecto, factores relacionados con el contrato, con el manejo del proyecto, los factores humanos y factores externos. 
Fueron encuestados noventa ingenieros para escuchar su opinión en torno a los grandes factores valorándolos en orden de importancia según su criterio se obtuvo que el factor de mayor importancia para los ingenieros es el factor del contrato con un 3.82 evaluado en una escala de 5, en segundo lugar se encuentra el factor del manejo de proyecto el que obtuvo un 3.59 en una escala de 5, luego con un 2.91 se encuentra el factor humano, luego le sigue el factor del proyecto con un promedio de 2.90 y por último, después de analizados, los resultados se obtuvo el factor externo con un 1.84 evaluado en una escala de 5 .

Después de realizadas estas encuestas y analizados los datos, se concluyó que los dos sub-factores de mayor impacto son "cumplimiento con las especificaciones de calidad requeridas" y "velar por el cumplimiento de la planificación durante el proyecto", no obstante, no están siendo bien gestionados.

Por último, se realizó una comparación entre la opinión de los clientes y los ingenieros.

Los clientes generales seleccionaron el sub-factor "cumplimiento con las especificaciones de calidad requeridas" como el de mayor importancia con un promedio de 3.71 en un máximo de 4 , le sigue "cumplimiento con el presupuesto acordado" con un promedio de 3.49 evaluado en un máximo de 4 y, en tercer lugar, se encuentra "cumplimiento de los códigos y reglamentos del Estado" con un promedio de 3.028.

Por su parte, los representantes de bienes raíces dieron a entender que en primer lugar se encuentran "cumplimiento con las especificaciones de calidad requeridas" y "cumplimiento con los códigos y reglamentos de Medio Ambiente" ambos con un promedio de 3.857 evaluados en un máximo de 4 , y le siguen "cumplimiento con el tiempo de entrega requerido" y "cumplimiento de los códigos y reglamentos del Estado” con un promedio de 3.57 de 4. 
Por otro lado, los clientes de grandes empresas manifestaron que para ellos el sub-factor "cumplimiento con las especificaciones de calidad requeridas" es el de mayor importancia con un promedio de 3.714 evaluado en un máximo de 4, le siguen "cumplimiento con el presupuesto acordado" y "elaboración de una planificación apropiada para el proyecto" con 3.286 de promedio y, por último, "cumplimiento con el tiempo de entrega requerido" con un promedio de 3.143 de 4 .

\section{Recomendaciones}

Se debe realizar una planificación completa y correcta para la realización de la obra teniendo en cuenta las pautas para la elaboración de la misma ya planteadas anteriormente.

A nuestro juicio, conviene poseer una política de calidad que asegure que cada actividad que se lleve a cabo dentro del proyecto tenga las especificaciones requeridas que han de ser seguidas con la mayor rectitud posible y así estar más seguro de que lo que se acepta es lo esperado.

Especificar con mayor detalle los contratos, atendiendo a entrevistas con los clientes para evaluar más claramente su visión y con esto presentarles una idea clara de lo que se realizara para evitar disyuntivas.

Es preciso verificar que todas las especificaciones expuestas en el contrato sean debidamente construidas y consultar con el cliente si el proceso va en la dirección conceptuada en el mismo.

Conviene realizar futuras investigaciones acerca de cómo se están gestionando los demás facto-res en el país.

Conviene realizar investigaciones cuantitativas para determinar cómo afectan en el presupuesto y en la duración del proyecto los factores reconocidos como más importantes. 
Camila Martinez, Samuel Morla, Nelson Peña, Richard Rosario, Carolina Slaiman, Heine Solis, Damaso V aldez, Coral Vargas

\section{Referencias}

Atkinson, R. (1999). Project management: cost, time and quality, two best guesses and a phenomenon, its time to accept other success criteria. International Journal of Project Management. 17(6), 337-342.

Belassi, W. \& Tuckel, O. I. (1996). A New Framework for Determining Critical Success/Failure Factors in Project. International Journal of Project Management, 14(3), 141-151.

Bilbao A., L., Campero Q., M. \& García de la Pastora C., D. G. (1987). Contratos de grandes obras. Revista de Ingeniería de Construcción, (2), 1-15.

Boyd, D. (2006). Understanding the Construction Client. Oxford: Blackwell.

Burdge, R. J. (2004). The concepts, process, and methods of social impact assesment. Middleton, WI: Social Ecology Press.

Campero, M. (2013). Rol de los principios de administración de proyectos en el manejo de obras civiles. Ingeniería de Construcción, 28(1), 81-94.

Campero, M. \& Bilbao, L. (1987). Contratos de grandes obras (Temas de reflexión). Revista de Ingeniería de Construcción, (2), 1-15.

Chan, A. P., Scott, D. \& Chan, A. P. (2004). Factors Affecting the Success of a Construction Project. Journal of Construction Engineering and Management, 130(1), 153-155.

Chan, A. P., Scott, D. \& Chan, A. (2004). Factors Affecting the Success of a Construction Project. Journal of Construction engineering and Management, 130(1), 153-155. 
Cheong Yong, Y. \& Mustaffa, N. E. (2011). Clients, Consultants and Contractors' perception of critical success factors for construction projects in Malasya. (Conferencia de Association of Researchers in Construction Management. Malasya).

Gambatese, J. B. (2008). Design's role in construction accident causality and prevention: Perspectives form an expert panel. Safety Science, 46(4), 675-691.

Hatush, K. \& Skitmore, M. (1997). Evaluating Contractor Prequalification Data: selection criteria and project success factors. Brisbane, Australia: Construction Management and Economics.

Hills, M. J., Fox, P. W. et al. (2008). The Role of Project Managers in Construction Industry Development. (In Bridge, Carolyn, Eds. $52^{\circ}$ ). Reunión Internacional Anual y 6th Congreso Mundial de Costos de Ingeniería, Manejo de Proyecto y Cantidad Encuestada. Congreso llevado a cabo en Toronto, Ontario, Canadá.

Hwang, B.-G. \& Janicia Lim, E.-S. (2013). Critical Success Factors for Key Project Players and Objectives: Case Study of Singapore. Journal of Construction Engineering and Management. 139(2), 204-215.

Jari, A. \& Bhangale, P. (2013). To Study Critical Factors Necessary for a Successful Construction Project. International Journal of Innovative Technology and Exploring Engineering. 2(5), 331-335.

Jha, K. N. (2013). Determinants of Construction Project Success in India. New Dehli: Springer.

Lim. C. S. \& Mohamed, M. Z. (1999). Criteria of project success: An exploratory re-examination. International Journal of Project Management, 17(4), 243-248.

Ministry of Finance Bangladesh. (2005). General Financial Rule. Recuperado de http:/ finmin.nic.in/gfr2005.pdf 
Moore, P. \& Riley, M. (2012). International Constructions Costs: A changing world academy. Londres: Arcadis.

Neringa Gudienè, L. R. (2013). An Evaluation of Critical Success Factors for Construction Projects using Expert Judgment. (1st International Virtual Scientific Conference. Vilnius, Lithuania).

Odesola, I. A., Otali, M. \& Ikediashi, D. I. (2013). Effects of ProjectRelated Factors on Construction Labour Productivity in Bayelsa State of Nigeria. Ethiopian Journal of Environmental Studies and Management, 6(6), 821.

Pakseresht, A., \& Asgari, G. (2012). Determining the Critical Success Factors in Construction Projects: AHP Approach. Interdisciplinary Journal of Contemporary Research in Business, 4(8), 383-393.

Pilar, F., Paiva, A., Domínguez, C. \& Varajão, J. (2009). Principais causas de atrasis di olano de trabalho de uma obra de construção civil. Covilhã: Inovação e Desenvolvimento.

Queensland University of Technology. (2001). Framework for measuring Success of Construction Project. Brisbane, Australia: PC Chan.

Tabish, S. S. (2011). Identification and evaluation of success factors for public construction projects. Recuperado http://www.tandfon line.com/doi/abs/10.1080/01446193.2011.611152\#.VpowPnhDcs

Thrum, D. (2005). Por qué una empresa debería tomar el control de sus proyectos de edificación. Harvard business review, 83(10), 1-9.

Universidad Autónoma Metropolitana. (2005). El factor bumano y su incidencia en elproyecto de construcción. Administración y tecnología para el diseño. Ciudad de México, México: Cervantes Abarca. 
University of Texas. (2006). Quantification of transactional dispute resolution costs for the U.S. construction industry. Texas: R. Gebken.

Valdes Vásquez, R. (2013). Social Sustainability Considerations during Planning and Design: Framework of Processes for Construction Projects. Journal of Construction Engineering \& Management, 139(1), 80-89.

Varajão, J. D. (2014). Critical Success Aspects in Project Management: Similarities And Differences Between The Construction And The Software Industry. Tebnicki Vjesnik/Technical Gazette, 21(3), 583-589.

Walker, D. H. (1995). An Investigation into Construction Time Performance. Construction Management and Economics, 13(3), 263-274.

Yang, J., Qiping Shen, G., Drew, D. \& Ho, M. (2010). Critical Success Factors for Stakeholder Management: Construction Practitioners' Perspectives. Journal of Construction Engineering and Management, 136(7), 778-786. 


\section{Camila Martínez}

Ingeniera egresada del Instituto Tecnológico de Santo Domingo (INTEC). Correo electrónico: camilaastwood@gmail.com

\section{Samuel Morla}

Ingeniero egresado del Instituto Tecnológico de Santo Domingo (INTEC). Correo electrónico: samuel_morla@hotmail.com

\section{Nelson Peña}

Ingeniero egresado del Instituto Tecnológico de Santo Domingo (INTEC). Correo electrónico: nelsonjp15@gmail.com

\section{Richard Rosario}

Ingeniero egresado del Instituto Tecnológico de Santo Domingo (INTEC). Correo electrónico: richard.rosario93@gmail.com

\section{Carolina Slaiman}

Ingeniera egresada del Instituto Tecnológico de Santo Domingo (INTEC). Correo electrónico: carolina.slaiman@gmail.com

\section{Heine Solis}

Ingeniera egresada del Instituto Tecnológico de Santo Domingo (INTEC). Correo electrónico: heinesolis@hotmail.com 
Estudio de los factores críticos para el éxito en la construcción de edificaciones en la ciudad de Santo Domingo, República Dominicana al año 2014

\section{Damaso Valdez}

Ingeniero egresado del Instituto Tecnológico de Santo Domingo (INTEC). Correo electrónico: damasovaldez16@gmail.com

\section{Coral Vargas Holguín}

Ingeniera egresada del Instituto Tecnológico de Santo Domingo (INTEC). Correo electrónico: coral.holguin@hotmail.com

Recibido: 27-09-2015

Aprobado: 22-10-2015 
